\title{
Multi Ball Training Method: A New Attempt of Table Tennis Training in Colleges and Universities
}

\author{
Wei Zheng ${ }^{1, a}$, Keyi Jin $^{1, \text { a }}$ \\ ${ }^{1}$ Dalian University, DaLian, Liaoning, 116622 \\ ${ }^{a}$ email:
} Keywords: Multi-ball Training Method, Universities and Colleges, Table Tennis, Experimental
Study

\begin{abstract}
Multi-ball training is a very effective method for table tennis training, which plays an important role in improving students' sports skills. Based on the author's coaching experience for many years, this paper uses documentation, experiment, mathematical statistics, expert interviews and other research methods to record the bat frequency of multi-ball training and single ball training in College Table Tennis class with the same training content and the same unit of time. In addition, record heart rate and recovery heart rate before and in training. Finally, make a comparison and analysis in the resulting data to assess amount and effect of the training.
\end{abstract}

\section{Introduction}

Multi-ball training in table tennis is an effective training method. Multi-ball training with different means of rotation, strength, speed, placement, arcs, combinations of different technologies and continuous ball hitting can compensate for the fewer to-and-fro times, more space and other weaknesses, in order to improve the exercise efficiency and make athletes grasp and strengthen a variety of difficult movements. Secondly, continuous hitting in multi-ball training can effectively increase the density and strength of the training, which not only strengthens the improvement of techniques and tactics, but also plays a role in training athletes' quality and will. With the ITTF introducing a series of reforms, it provides some new requirements of table tennis training and multi-ball training, as a focus of training, should also follow it to make appropriate adjustments in order to better serve our training to improve athletes’ techniques and tactics to a greater degree.

\section{Research Subjects and Methods}

Research Subjects. Experimental method: 10 students in table tennis special project class of 2012 to 2015 in some physical culture institute. Questionnaire survey: 50 students majoring in Tennis in Physical Education in Dalian University。

Research Methods. Documentation: search and collect literature on multi-ball training in table tennis in domestic sports journals by hand or computer to provide a theoretical basis of this thesis.

Experimental method. Select 10 students randomly and choose the same training content and the same training time to record athletes' heart rate before, in and after the multi-ball training and single ball training. Heart rate before exercise refers to heart beats per minute in quiet situation. Exercise heart rate after exercise is instant heart rate, which is equal to beats in 10s multiplied by 6. Recovery heart rate is beats in 10s multiplied by 6 after a 3-minute rest. At the same time, it is necessary to record the hitting times.

Questionnaire. Randomly select 50 students to make a questionnaire study about the features, effect application of multi ball training method and the result in practice of different athletes and coaches. 


\section{Results and Discussion}

An Analysis of influence of Multi Ball Training Method on Athletes' Training Intensity and Density. In table tennis practical training, a variety of technical training can adopt to multi-ball training method. In this paper, the experiment measures heart rate before the multi-ball training, heart rate after exercise, recovery heart rate and the hitting times. Table 1 follows:

Table 1 Heart rate statistical table in different phases in multi ball training

\begin{tabular}{|c|c|c|c|c|c|c|c|c|}
\hline number & $\begin{array}{c}\text { before } \\
\text { multi } \\
\text { ball } \\
\text { training }\end{array}$ & $\begin{array}{c}\text { before } \\
\text { single } \\
\text { ball } \\
\text { training }\end{array}$ & $\begin{array}{l}\text { in multi } \\
\text { ball } \\
\text { training }\end{array}$ & $\begin{array}{c}\text { in single } \\
\text { ball } \\
\text { training }\end{array}$ & $\begin{array}{l}\text { recovery } \\
\text { in multi } \\
\text { ball } \\
\text { training }\end{array}$ & $\begin{array}{l}\text { recovery } \\
\text { in single } \\
\text { ball } \\
\text { training }\end{array}$ & $\begin{array}{l}\text { hitting } \\
\text { times in } \\
\text { multi } \\
\text { ball } \\
\text { training }\end{array}$ & $\begin{array}{l}\text { hitting } \\
\text { times in } \\
\text { single } \\
\text { ball } \\
\text { training }\end{array}$ \\
\hline 1 & 72 & 72 & 192 & 132 & 90 & 78 & 238 & 152 \\
\hline 2 & 70 & 69 & 198 & 126 & 84 & 72 & 241 & 148 \\
\hline 3 & 69 & 67 & 192 & 138 & 90 & 66 & 249 & 162 \\
\hline 4 & 70 & 71 & 192 & 132 & 84 & 72 & 228 & 142 \\
\hline 5 & 72 & 72 & 186 & 132 & 90 & 66 & 234 & 158 \\
\hline 6 & 73 & 71 & 192 & 138 & 90 & 72 & 223 & 150 \\
\hline 7 & 69 & 71 & 186 & 132 & 78 & 72 & 220 & 162 \\
\hline 8 & 68 & 69 & 192 & 136 & 84 & 72 & 238 & 154 \\
\hline 9 & 69 & 68 & 180 & 120 & 90 & 66 & 218 & 159 \\
\hline 10 & 72 & 71 & 192 & 138 & 84 & 78 & 236 & 162 \\
\hline $\begin{array}{c}\text { average } \\
\text { value }\end{array}$ & 70 & 69 & 190 & 131 & 86 & 71 & 232 & 155 \\
\hline
\end{tabular}

Compare the load in forehand walk multi ball training and one to one single ball training with the same training content and training time $(5 \mathrm{~min})$ and the experiment shows that heart rate after exercise in multi ball training is significantly higher than that in one to one single ball training. From Table 1, it can be seen that multi-ball training has greater density and intensity in equal time in pulling the ball. Specifically show that the heart rate is almost the same before training and after training, the average heart rate in multi-ball training is 190 and the single ball training is 131, which is 59 less. Recovery heart rate in multi ball training is 15 more than single ball training. Recovery heart rate after exercise in multi ball training is $82 \%$ of that before training, which fully explains the intensity of multi ball training is much greater than single-ball ball exercise and the recovery is also faster; hitting times in single ball training is 77 times less than that in multi ball training, which means the density of multi ball training is $50 \%$ higher. It is useful for an athlete to complete the training mission in the effective training time and improve the competitive level.

The results show that the multi ball training is not only helpful to the establishment and consolidation of the basic technical movements, but also to increasing the exercise load and improving the function of the cardiovascular and respiratory systems. Therefore, we learn that multi ball training can increase ball speed, hitting power, the difficulty of movement and athletes need to continuously move fast with great density and strength. Due to the slower and more stable movement in single ball training method, athletes have sufficient time to hit the ball. Therefore, in order to improve the ability in hypoxia, we must find a method in accordance with the rhythm of table tennis, which can combine technical movements together. Multi ball training is just an ideal method for this requirement.

The Application of Tactical Combination in Multi Ball Training. When do tactical training for athletes, multi-ball training is a good choice. In the technical combination training in table tennis, the quality of training and the results in some technical training content with single ball training methods and means are not ideal. It is susceptible to opponents' ball quality and cannot reach the 
training objectives and requirements. For example, when you pull straight forehand and hit the ball continuously, if you use the single ball training method, the ladder player cannot master well and it is easy to clean the ball. In addition, the ball is not high quality which will affect the continuity and exercise difficulty. However, these problems do not exist in multi ball training, which ensures the quality and intensity of training. In the current national team, using multi ball training method to develop athletes' tactical characteristics is common. In multi-ball technical and tactical training, we should control the training rhythm between different groups and we cannot hit the ball continuously with the same rhythm. In this case, we often use interval training to train within 15 minutes in several groups. There is a group pause about 30 seconds based on athletes' circumstances with a two-second pause between different groups. In a tactical combination, we should accelerate the speed as much as possible in accordance with the tactical set and acceptable range for athletes, in order to improve athletes' response threshold to adapt to fast ball speed in actual games.

An Analysis of the Function of Multi Ball Training in Table Tennis. One is the formation of movements and correction of the techniques. It shows in psychology and physiology research that within a certain range, the stronger the conditioned stimulus and the unconditioned stimulus, the more likely to establish a conditioned reflex. The density and intensity of multi ball training is stronger than the single ball training. Within a certain time, practice more often with coaches' tips and it is easier for athletes to correct movement techniques. Second is to strengthen the footwork exercises. Due to the good continuity in multi ball training, trainees can run continuously. For example: when practice the above in the full court, the trainee may run continuously for about 30 balls. This is difficult to achieve in the single ball training. In addition, multi-ball training can practice a variety of footwork, including front and rear footwork, left and right footwork, and some combination footwork. Based on each player' weaknesses, we can also make targeted exercise. Third, it could improve the combination ball techniques. High-level athletes increasingly focus on combination ball exercises and the role of combination ball in the game is also very significant. Using the Multi ball training method to practice combination techniques may increase plate times, which is fewer in the single ball training. It can also improve athletes' response ability. Use different placements and different speeds of the ball to improve the combination ball techniques. Fourth, the develop athletes' good will to never give up in the face of adversity. Moreover, they will do not relax at the lead and do not hesitate in the face of pressure. In the face of fatigue they can withstand it. All these are necessary qualities for modern table tennis athletes. After the implementation of 11 Points Competition System, it requires the athletes to have stronger mental qualities. In the multi ball training with high-intensity, high-density and highly complexity, it requires athletes to insist on that, struggle it and never give up easily. It is effective to exercise athletes' willpower.

\section{Conclusion and Suggestion}

Conclusion. The intensity of multi ball training is more than $45 \%$ higher than that in single ball training; the hitting time is $50 \%$ higher. The load of multi ball training is significantly greater than that in single ball training. Multi-ball training method has an obvious effect on improving players' technical and tactical levels, special qualities and will.

Suggestion. The correct allocation of multi ball training and single ball training. Multi ball training accounts for about $30 \%-40 \%$ of the total amount of training. This will ensure the effectiveness and improve player' specific endurance qualities. Make full preparations before the multi-ball training to avoid injury due to high density and high strength which may affect the normal training. Improve the quality of the ball in the placement, rotation, strength, arcs and other aspects which may well be related to a single ball as much as possible. Arrange multi ball training for one athlete according to the technical condition of the athletes to improve the combat ability of athletes. The exercise amount of each group should be based on athletes' age, gender, physical fitness and play features, which cannot be generalized. Use multi-ball training athletes to form players' different features rather than a uniform pattern. 


\section{References}

[1] Teng Shougang, Xu Linan. The Application of Multi-player Multi-ball Training Method to Table Tennis Teaching and Training [J]. Journal of Wuhan Institute of Physical Education, 2012, 11: 82-86.

[2] Liu Jin. A Study on the Multi-ball Training for table Tennis Beginners’ Technique Level Development [D]. Hunan Normal University, 2012.

[3] Xu Linan. The Application of Multi-ball Training Method to Table Tennis Combination Techniques and Tactics [D]. Wuhan Institute of Physical Education, 2012.

[4] Tang Yu. An Experimental Study on Multiplayer Multi-ball Training method in the Techniques and Tactics of Table Tennis [D]. Central China Normal University, 2014.

[5] Wang Xiao. An Experimental study on Multi-ball Training in Table Tennis Training [D]. Shandong Physical Education Institute, 2014. 